\title{
Resistência à Helicoverpa armigera em genótipos de tomateiro obtidos do cruzamento de Solanum lycopersicum com Solanum galapagense
}

\author{
Alex Antônio da Silva(1), Marcela Carvalho Andrade(1), Regis de Castro Carvalho( ${ }^{(1)}$, Irã Pinheiro Neiva ${ }^{(2)}$, \\ Daniela Costa Santos ${ }^{(2)}$ e Wilson Roberto Maluf(2)
}

(1)Universidade Federal de Lavras (Ufla), Departamento de Biologia, Campus Universitário, Caixa Postal 3037, CEP 37200-000 Lavras, MG,
Brasil. E-mail: alex.antonio.silva27@gmail.com, marcellinhaufla@gmail.com, regisccarvalho@hotmail.com (2)Ufla, Departamento de Agricultura,
Caixa Postal 3037, CEP 37200-000 Lavras, MG, Brasil. E-mail: iraagronomia@yahoo.com.br, dani_solos@hotmail.com, wrmaluf@dag.ufla.br

Resumo - O objetivo deste trabalho foi verificar a resistência à lagarta Helicoverpa armigera (Lepidoptera: Noctuidae), em genótipos de tomateiro obtidos do cruzamento interespecífico de Solanum lycopersicum x $S$. galapagense, e a associação dessa resistência com a densidade de tricomas glandulares do tipo IV. Genótipos da população $\mathrm{F}_{2}$, derivada do cruzamento $S$. lycopersicum TOM-684 x $S$. galapagense LA-1401, selecionados quanto à baixa e à alta densidade de tricomas glandulares, respectivamente, foram submetidos a dois testes de resistência à lagarta $H$. armigera em laboratório, um de antibiose e outro de antixenose. Os genótipos da população $\mathrm{F}_{2}$, com alta densidade de tricomas glandulares do tipo IV (BPX-486-17, BPX-486-62, BPX-486-10, BPX-486-46 e BPX-486-08), apresentam maiores níveis de resistência, tanto por antibiose quanto por antixenose, do que os genótipos com baixa densidade de tricomas glandulares (BPX-486-313 e BPX-486-383) e do que as testemunhas 'Santa Clara' e TOM-684. Os níveis de resistência foram menores do que os do genitor silvestre LA-1401.

Termos para indexação: manejo integrado de pragas, melhoramento de plantas, seleção indireta, tricomas foliares.

\section{Resistance to Helicoverpa armigera in tomato genotypes obtained from the crossing of Solanum lycopersicum x Solanum galapagense}

\begin{abstract}
The objective of this work was to verify the resistance to the Helicoverpa armigera (Lepidoptera: Noctuidae) caterpillar by tomato genotypes obtained from interspecific crossing of Solanum lycopersicum $\mathrm{x} S$. galapagense, and the association of this resistence with the density of type IV glandular trichomes. Genotypes of the $\mathrm{F}_{2}$ population, obtained from the crossing of $S$. lycopersicum TOM- 684 x S. galapagense LA-1401, selected for low and high densities of glandular trichomes, respectively, were subjected to two laboratory resistance tests, one for antibiosis and other for antixenosis. Genotypes of the $\mathrm{F}_{2}$ population, with high density of type IV glandular trichomes (BPX-486-17, BPX-486-62, BPX-486-10, BPX-486-46 and BPX-486-08), showed higher resistance levels, both by antibiosis and antixenosis, than the genotypes with low-density glandular trichomes (BPX-486-313 and BPX-486-383) and the witnesses 'Santa Clara' and TOM-684. Resistance levels were lower than those of the wild parent LA-1401.
\end{abstract}

Index terms: pest integrated management, plant breeding, indirect selection, leaf trichomes.

\section{Introdução}

Helicoverpa armigera (Hübner) (Lepidoptera: Noctuidae) é um inseto polífago e de ampla distribuição geográfica (Ali et al., 2009). Até meados de 2013, esta praga era considerada quarentenária no Brasil, ano em que houve a confirmação de sua ocorrência nas culturas da soja e do algodão nos estados de Goiás, Bahia e Mato Grosso, o que representa o primeiro registro de $H$. armigera no continente americano
(Czepak et al., 2013). Specht et al. (2013) ampliaram o registro de ocorrência e apresentaram a caracterização morfológica e molecular da praga. Recentemente, também foi relatada a ocorrência deste inseto em cultivos de tomate, no Estado do Espírito Santo (Pratissoli et al., 2015).

Lagartas recém-eclodidas de $H$. armigera alimentam-se de folhas e partes tenras do tomateiro até atingirem o terceiro instar ou até os cinco dias de idade. Em seguida, perfuram e penetram nos frutos através de 
galerias, para se alimentarem da placenta e de sementes. Nos orifícios feitos pelas lagartas, desenvolvem-se microrganismos que causam a podridão mole. Nesta fase, os prejuízos à cultura podem chegar a até $55 \%$ de perda da produção (Usman et al., 2013).

O controle químico é o principal método empregado contra pragas na cultura do tomateiro, em todo o mundo. No entanto, o uso de inseticidas como única ou principal tática de manejo pode ocasionar danos severos ao ambiente, como desequilíbrio biológico, e danos à saúde do trabalhador rural e do consumidor, além de aumento dos custos de produção (Silva et al., 2009; Mamta et al., 2016).

Para minimizar os problemas advindos do controle químico, têm-se buscado táticas de controle alternativas para uso no manejo integrado de pragas (MIP) da cultura. Entre estas, o uso de cultivares resistentes é considerado ideal, por ser de custo relativamente baixo, possibilitar o equilíbrio populacional dos insetos com seus inimigos naturais, não poluir o ambiente e, principalmente, não oferecer riscos à saúde humana (Talekar et al., 2006; Lucini et al., 2015).

Nos programas de melhoramento, tem-se buscado incorporar ao tomateiro cultivado a resistência a insetos, encontrada em acessos de espécies silvestres. Nessas espécies, os principais fatores de resistência são os tricomas glandulares e aleloquímicos, produzidos e exsudados pelos tricomas. Esses compostos têm sido associados aos mecanismos de resistência de antibiose e antixenose (Maluf et al., 2010; Tian et al., 2012; Dias et al., 2016).

Fontes de resistência à $H$. armigera já foram relatadas em acessos das espécies Solanum pennellii, $S$. habrochaites e $S$. pimpinellifolium (Simmons et al., 2004; Selvanarayanan \& Narayanasamy, 2006; Talekar et al., 2006; Safuraie-Parizi, et al., 2014; Muthukumaran, 2016). Além dessas, alguns acessos de $S$. galapagense, apesar de ainda não terem sido documentados como resistentes à $H$. armigera, são promissores, pois apresentam altos níveis de resistência a outros insetos que atacam o tomateiro, associados com a alta densidade de tricomas glandulares do tipo IV e à elevada concentração foliar do aleloquímico acilaçúcar (Jouy et al., 1992; Simmons \& Gurr, 2005; Firdaus et al., 2013; Lucatti et al., 2013; Andrade, 2015).

O objetivo deste trabalho foi verificar a resistência à lagarta Helicoverpa armigera (Lepidoptera:
Noctuidae), em genótipos de tomateiro obtidos do cruzamento interespecífico de Solanum lycopersicum $\mathrm{x} S$. galapagense, e a associação dessa resistência com a densidade de tricomas glandulares do tipo IV.

\section{Material e Métodos}

A partir do cruzamento interespecífico (TOM-684 x LA-1401), obteve-se uma população $\mathrm{F}_{2}$, denominada de BPX-486. TOM-684 é uma linhagem-elite de S. lycopersicum, pré-comercial, com baixa densidade de tricomas glandulares e baixo teor de acilaçúcares nas folhas (Maluf et al., 2010; Costa, 2013). LA-1401 é um acesso silvestre da espécie $S$. galapagense, com alta densidade de tricomas glandulares do tipo IV, alto teor de acilaçúcares nas folhas e resistente à mosca-branca Bemisia tabaci (Genn.) (Hemiptera: Aphididae) (Lucatti et al., 2013).

A partir dessa população, utilizaram-se 145 plantas, mais 10 plantas da cultivar 'Santa Clara', da linhagem TOM-684 e do acesso LA-1401. Todas foram avaliadas quanto à densidade (número médio por $\mathrm{mm}^{2}$ ) e tipos de tricomas presentes nas faces abaxial e adaxial dos folíolos, conforme Luckwill (1943) e Kraus \& Arduim (1997).

Após essa avaliação sete plantas foram selecionadas da população BPX-486, das quais duas com baixa e cinco com alta densidade de tricomas glandulares (Tabela 1), que representaram os dois extremos encontrados. Esses genótipos, juntamente com o acesso LA-1401, foram clonados por meio de estaquia dos brotos axilares para multiplicação (Gonçalves et al., 2006).

Aproximadamente 30 dias após o início do enraizamento dos clones e semeadura das testemunhas 'Santa Clara' e TOM-684, em bandejas de poliestireno expandido de 72 células, realizou-se o transplante de 18 mudas de cada genótipo em vasos de polietileno, com capacidade para $5 \mathrm{~L}$ de substrato, que continha terra, areia e o composto Provaso à proporção 3:1:1, em casa de vegetação, para utilização nos testes de resistência à lagarta $H$. armigera. Os tratos culturais utilizados na condução das plantas foram realizados conforme os preconizados para a cultura do tomateiro (Alvarenga, 2013).

Para o início da criação de manutenção no laboratório, pupas de $H$. armigera foram obtidas 
de uma criação massal (Bug Agentes Biológicos, Piracicaba, SP, Brasil).

As pupas foram sexadas com auxílio de microscópio estereoscópico e transferidas para gaiolas de tubos de PVC - com cerca de $30 \mathrm{~cm}$ de altura e $10 \mathrm{~cm}$ de diâmetro -, revestidas interiormente com papel-toalha e fechadas com tecido voil. Em cada gaiola, foram colocados aproximadamente 20 casais de $H$. armigera .

Após a emergência dos adultos, foram colocados dois tubos de vidro - de $1,0 \mathrm{~cm}$ de diâmetro e $3,0 \mathrm{~cm}$ de altura - com solução de mel a $10 \%$, embebida em algodão, para a alimentação dos adultos. O papel-toalha com os ovos das mariposas foram retirados das gaiolas e mantidos em placas de Petri, de $20 \mathrm{~cm}$ de diâmetro, até a eclosão das lagartas.

As lagartas foram mantidas em tubos de ensaio - de $15 \mathrm{~cm}$ de altura e $1,5 \mathrm{~cm}$ de diâmetro -, que continham dieta artificial preparada de acordo com Greene et al. (1976). A temperatura da sala de criação foi mantida a $25 \pm 2^{\circ} \mathrm{C}$, umidade relativa de $70 \pm 10 \%$ e fotófase de 12 horas.

Os testes de sobrevivência e antixenose (não preferência) de $H$. armigera foram realizados, com os genótipos da população de tomate BPX-486, selecionados quanto à alta e à baixa densidades de tricomas glandulares, conforme metodologia já descrita, juntamente com o acesso LA-1401 e as testemunhas 'Santa Clara' e TOM-684. Cinco lagartas recém-eclodidas, com idade inferior a 24 horas, provenientes da criação de manutenção, foram liberadas em placas de Petri ( $5 \mathrm{~cm}$ de diâmetro) forradas com papel-filtro umedecido com água destilada, que continham um folíolo correspondente a cada genótipo (com a face abaxial voltada para cima), coletado no terço superior das plantas dos genótipos de tomateiro, que estavam com 40 dias após o transplante das mudas para os vasos. As placas foram fechadas com filme de PVC perfurado com alfinete entomológico e mantidas em câmara climatizada do tipo $\operatorname{BOD}\left(25 \pm 2^{\circ} \mathrm{C}\right.$, umidade relativa de $70 \pm 10 \%$ e fotófase de 12 horas). Utilizou-se o delineamento experimental inteiramente casualizado, com 10 tratamentos e 18 repetições.

Avaliou-se a sobrevivência das lagartas 24, 48 e 72 horas após a liberação, por meio da contagem de lagartas vivas sobre os folíolos dos diferentes genótipos de tomateiro.

$O$ teste de antixenose, em condições de livre escolha para alimentação, foi montado aos 35 dias após o transplante das mudas para os vasos. Para isso, folíolos do terço superior das plantas de tomateiro, de tamanhos semelhantes, foram destacados com o auxílio de tesoura e colocados em sacos de papel, com a devida identificação do genótipo e da repetição. No laboratório, o material vegetal foi imediatamente colocado em placas de Petri de $15 \mathrm{~cm}$ de diâmetro, forradas com papel filtro umedecido com água destilada. Os folíolos foram dispostos em círculo, com a face abaxial voltada para cima, equidistantes entre si, formando uma arena. Cada placa continha, assim, um folíolo de cada um dos sete genótipos testados. No centro de cada placa foram liberadas 24 lagartas de H. armigera, com quatro dias de idade, provenientes da criação de manutenção. Antes da liberação, as lagartas permaneceram seis horas sem alimentação. As placas foram mantidas em câmara climatizada do tipo BOD $\left(25 \pm 2^{\circ} \mathrm{C}\right.$, umidade relativa de $70 \pm 10 \%$ e fotófase de

Tabela 1. Densidade de tricomas glandulares dos tipos IV e (I+VI+VII) sobre as faces abaxial (ab) e adaxial (ad) dos genótipos de tomateiro selecionados e classificação quanto à densidade de tricomas do tipo IV ${ }^{(1)}$.

\begin{tabular}{|c|c|c|c|c|c|c|}
\hline \multirow[t]{2}{*}{ Genótipos } & \multicolumn{4}{|c|}{ Densidade de tricomas glandulares (média \pm erro padrão do número por mm²) } & \multirow[t]{2}{*}{ Total } & \multirow{2}{*}{$\begin{array}{c}\text { Densidade de } \\
\text { tricomas do tipo IV }\end{array}$} \\
\hline & IV, face abaxial & I+VI+VII, face abaxial & IV, face adaxial & I+VI+VII, face adaxial & & \\
\hline 'Santa Clara' & $0,0 \pm 0,00 \mathrm{c}$ & $0,0 \pm 0,00 \mathrm{a}$ & $0,0 \pm 0,00 \mathrm{~b}$ & $0,0 \pm 0,00 \mathrm{a}$ & $0,0 \pm 0,00 \mathrm{c}$ & baixa \\
\hline TOM-684 & $0,0 \pm 0,00 \mathrm{c}$ & $0,0 \pm 0,00 \mathrm{a}$ & $0,0 \pm 0,00 \mathrm{~b}$ & $0,8 \pm 0,46 \mathrm{a}$ & $0,8 \pm 0,46 \mathrm{c}$ & baixa \\
\hline BPX-486-313 & $0,0 \pm 0,00 \mathrm{c}$ & $0,0 \pm 0,00 \mathrm{a}$ & $0,0 \pm 0,00 \mathrm{~b}$ & $0,3 \pm 0,13 \mathrm{a}$ & $0,3 \pm 0,13 \mathrm{c}$ & baixa \\
\hline BPX-486-383 & $0,0 \pm 0,00 \mathrm{c}$ & $0,0 \pm 0,00 \mathrm{a}$ & $0,0 \pm 0,00 \mathrm{~b}$ & $0,0 \pm 0,00 \mathrm{a}$ & $0,0 \pm 0,00 \mathrm{c}$ & baixa \\
\hline BPX-486-17 & $43,0 \pm 5,51 \mathrm{a}$ & $0,0 \pm 0,00 \mathrm{a}$ & $5,0 \pm 3,52 \mathrm{a}$ & $0,3 \pm 0,13 \mathrm{a}$ & $48,3 \pm 9,16 \mathrm{a}$ & alta \\
\hline BPX-486-62 & $21,1 \pm 3,84 b$ & $0,0 \pm 0,00 \mathrm{a}$ & $8,3 \pm 0,57 \mathrm{a}$ & $0,3 \pm 0,18 \mathrm{a}$ & $29,7 \pm 4,59 b$ & alta \\
\hline BPX-486-10 & $16,7 \pm 2,60 b$ & $0,0 \pm 0,00 \mathrm{a}$ & $8,0 \pm 4,51 \mathrm{a}$ & $0,6 \pm 0,23 \mathrm{a}$ & $25,3 \pm 7,34 b$ & alta \\
\hline BPX-486-46 & $33,3 \pm 8,41 \mathrm{a}$ & $0,0 \pm 0,00 \mathrm{a}$ & $5,0 \pm 1,99 a$ & $0,0 \pm 0,00 \mathrm{a}$ & $38,3 \pm 10,4 a$ & alta \\
\hline BPX-486-08 & $32,3 \pm 6,64 a$ & $0,0 \pm 0,00 \mathrm{a}$ & $4,3 \pm 1,33 \mathrm{a}$ & $0,0 \pm 0,00 \mathrm{a}$ & $36,6 \pm 7,97 b$ & alta \\
\hline LA-1401 & $20,3 \pm 1,66 b$ & $0,0 \pm 0,00 \mathrm{a}$ & $7,1 \pm 2,00 \mathrm{a}$ & $0,2 \pm 0,11 \mathrm{a}$ & $27,4 \pm 3,77 b$ & alta \\
\hline
\end{tabular}

${ }^{(1)}$ Médias seguidas de letras iguais, nas colunas, pertencem ao mesmo grupo, pelo teste de Scott-Knott, a 5\% de probabilidade. 
12 horas). $\mathrm{O}$ ensaio foi montado em delineamento de blocos ao acaso, com 7 tratamentos e 18 repetições.

As avaliações foram realizadas 12, 24, 36 e 48 horas após a liberação dos insetos, pela contagem de lagartas presentes sobre cada folíolo de tomateiro. Também foi avaliada a percentagem de área foliar consumida, por meio de notas visuais aos danos (Boiça Junior et al., 2013), após a última avaliação da contagem de lagartas.

Os dados da densidade de tricomas e de antixenose foram transformados em $(x+0,5)^{1 / 2}$, enquanto os dados de sobrevivência e da nota visual de consumo foliar em arco-seno $(\mathrm{x} / 100)^{1 / 2}$, antes de se proceder à análise de variância. As médias foram agrupadas pelo teste de Scott-Knott, a 5\% de probabilidade, por meio do aplicativo estatístico Sisvar (Universidade Federal de Lavras, Lavras, MG). Realizaram-se, também, as correlações entre as densidades do tricoma glandular predominante (tipo IV) da face abaxial dos folíolos, dos genótipos com a sobrevivência, antixenose e nota de consumo foliar da lagarta $H$. armigera, por meio do pacote computacional SAS (SAS Institute Inc., Cary, NC, EUA).

\section{Resultados e Discussão}

Asobrevivência da lagarta $H$. armigera, 24 horas após a liberação nas placas de Petri, foi significativamente menor sobre os genótipos de tomateiro selecionados quanto à alta densidade de tricomas glandulares do tipo IV, bem como sobre o acesso LA-1401 (em média 22,2\% menor), em comparação aos genótipos selecionados quanto à baixa densidade desse tipo de tricoma e com as testemunhas 'Santa Clara' e TOM-684 (Tabela 2).

Verificou-se, também, menor sobrevivência de $H$. armigera mantida sobre os folíolos dos genótipos BPX-486, com alta densidade de tricomas glandulares do tipo IV, após 48 e 72 horas da liberação das lagartas nas placas (em média 54,5\% menor), em comparação aos genótipos com baixa densidade de tricomas glandulares. Contudo, a sobrevivência de $H$. armigera foi maior sobre esses cinco genótipos da população BPX-486, com alta densidade de tricomas glandulares do tipo IV, comparados ao genótipo LA-1401. Neste genótipo, houve uma redução da média de sobrevivência de $84,1 \%$, após 48 e 72 horas, em comparação aos genótipos selecionados quanto à baixa densidade de tricomas glandulares do tipo IV e às testemunhas comerciais (Tabela 2).

As correlações entre a sobrevivência de $H$. armigera e a densidade de tricomas glandulares do tipo IV, da face abaxial dos folíolos dos genótipos, foram altas, significativas e negativas, nos períodos de 24, 48 e 72 horas após a liberação das lagartas, o que evidencia que os tricomas foliares do tipo IV exercem um importante papel no aumento dos níveis de antibiose do tomateiro a lagartas de $H$. armigera (Tabela 2).

Os genótipos BPX-486-08, BPX-486-17 e BPX-486-46 apesar de apresentarem, em média, densidade de tricomas glandulares do tipo IV superior a do acesso LA-1401, na face abaxial dos folíolos (Tabela 1), possivelmente, têm teores intermediários de acilaçúcares nas folhas, em comparação ao acesso LA-1401, que apresenta alto teor (Lucatti et al., 2013), e aos genótipos 'Santa Clara' e TOM-684, que apresentam baixos teores de acilaçúcares (Maluf et al., 2010). Isso poderia explicar o fato de os genótipos selecionados quanto à alta densidade de tricomas glandulares do tipo IV terem apresentado o mesmo nível de antibiose à lagarta que o acesso silvestre LA-1401, na primeira avaliação, realizada 24 horas após a liberação das lagartas, apesar de terem mostrado menores níveis de resistência que o LA-1401 nas

Tabela 2. Sobrevivência (média \pm erro padrão) e correlação de lagartas recém-eclodidas de Helicoverpa armigera, às 24, 48 e 72 horas após a liberação sobre a face abaxial dos folíolos de genótipos de tomateiro com diferentes densidades de tricomas glandulares do tipo IV (temperatura, $25 \pm 2^{\circ} \mathrm{C}$; umidade relativa do ar, $70 \pm 2 \%$; e fotófase de 12 horas) $)^{(1)}$.

\begin{tabular}{lcccc}
\hline Genótipo & \multirow{2}{*}{$\begin{array}{c}\text { Densidade de tri- } \\
\text { comas do tipo IV }\end{array}$} & \multicolumn{3}{c}{ Sobrevivência (\%) } \\
\cline { 3 - 5 } & baixa & $94,4 \pm 2,17 \mathrm{a}$ & $88,8 \pm 3,32 \mathrm{a}$ & $60,0 \pm 4,27 \mathrm{a}$ \\
'Santa Clara' & baixa & $85,5 \pm 2,70 \mathrm{a}$ & $71,1 \pm 3,69 \mathrm{a}$ & $51,1 \pm 4,63 \mathrm{a}$ \\
TOM-684 & baixa & $84,4 \pm 3,45 \mathrm{a}$ & $67,7 \pm 3,66 \mathrm{a}$ & $46,6 \pm 3,96 \mathrm{a}$ \\
BPX-486-313 & baixa & $82,2 \pm 3,57 \mathrm{a}$ & $70,0 \pm 4,35 \mathrm{a}$ & $46,6 \pm 4,57 \mathrm{a}$ \\
BPX-486-383 & alta & $71,1 \pm 4,91 \mathrm{~b}$ & $38,8 \pm 6,15 \mathrm{~b}$ & $20,0 \pm 5,11 \mathrm{~b}$ \\
BPX-486-17 & alta & $68,8 \pm 4,34 \mathrm{~b}$ & $42,2 \pm 3,18 \mathrm{~b}$ & $21,1 \pm 4,70 \mathrm{~b}$ \\
BPX-486-62 & alta & $68,8 \pm 5,41 \mathrm{~b}$ & $37,7 \pm 3,57 \mathrm{~b}$ & $16,6 \pm 3,33 \mathrm{~b}$ \\
BPX-486-10 & alta & $66,6 \pm 4,27 \mathrm{~b}$ & $31,1 \pm 3,69 \mathrm{~b}$ & $18,8 \pm 3,41 \mathrm{~b}$ \\
BPX-486-46 & alta & $65,5 \pm 3,89 \mathrm{~b}$ & $42,2 \pm 5,33 \mathrm{~b}$ & $16,6 \pm 3,70 \mathrm{~b}$ \\
BPX-486-08 & alta & $63,3 \pm 4,91 \mathrm{~b}$ & $18,8 \pm 4,70 \mathrm{c}$ & $1,1 \pm 1,11 \mathrm{c}$ \\
LA-1401 & & & & \\
Correlação (r) & & $-0,81^{*}$ & $-0,78^{*}$ & $-0,79 *$ \\
Densidade x sobrevivência & & &
\end{tabular}

${ }^{(1)}$ Médias seguidas de letras iguais, nas colunas, pertencem ao mesmo grupo, pelo teste de Scott-Knott, a $5 \%$ de probabilidade. *Significativo a $5 \%$, pelo teste de F. 
demais avaliações, realizadas às 48 e 72 horas após a liberação das lagartas.

A baixa sobrevivência também foi observada em lagartas recém-eclodidas de $H$. armigera, sobre os folíolos de acessos dos seguintes tomateiros: $S$. pennellii e $S$. habrochaites (Simmons et al., 2004); LA-716 e LA-1777 (S. habrochaites var. hirsutum) (Talekar et al., 2006); em acessos de S. lycopersicum; e num híbrido de tomateiro com $S$. pimpinellifolium (Selvanarayanan \& Narayanasamy, 2006). Todos esses genótipos apresentavam alta densidade de tricomas glandulares.

A ação dos tricomas glandulares do tipo IV, sobre o aumento da resistência por antibiose em tomateiro, também tem sido detectada em outros insetos-praga da cultura. Moreira et al. (2009) observaram que a sobrevivência de lagartas recém-eclodidas de Tuta absoluta (Meyrick) (Lepidoptera: Gelechiidae), na segunda geração, atingiu apenas $10 \%$, quando mantida sobre o acesso PI-134417 de S. habrochaites var. glabratum, que apresenta alta densidade de tricomas glandulares do tipo IV nas folhas.

Genótipos $\mathrm{F}_{2}$ selecionados quanto à alta densidade de tricomas glandulares do tipo IV, obtidos do cruzamento de tomateiro com o acesso LA-716 (S. pennellii), que assim como o LA-1401 ( $S$. galapagense) apresenta altos teores foliares de acilaçúcares, mostraram efeito antibiótico no desenvolvimento do ácaro Tetranychus urticae (Koch) (Acari: Tetranychidae) (Lucini et al., 2015).
Em todas as avaliações de antixenose, após a liberação das lagartas de $H$. armigera com quatro dias de vida, observou-se número significativamente menor desses insetos sobre os folíolos dos genótipos de tomateiro, selecionados quanto à alta densidade de tricomas do tipo IV, e sobre o acesso LA-1401 (em média $69,9 \%$ menor), comparados aos genótipos com baixa densidade de tricomas do tipo IV (Tabela 3). Além disso, os genótipos selecionados quanto à alta densidade de tricomas do tipo IV apresentaram significativamente menores notas visuais de consumo do que os genótipos com baixa densidade de tricomas do tipo IV, e notas maiores do que as do acesso LA-1401.

As correlações lineares do número de lagartas e da nota visual de consumo foliar com a densidade de tricomas glandulares do tipo IV, da face abaxial dos folíolos dos genótipos, foram todas altas e negativas (Tabela 3). Esses resultados mostram a ocorrência da associação entre a maior densidade de tricomas glandulares do tipo IV, sobre os folíolos dos genótipos de tomateiro, e o maior nível de resistência do tipo antixenose quanto à alimentação de lagartas de H. armigera.

Os genótipos de tomateiro selecionados quanto à alta densidade de tricomas glandulares do tipo IV mostraram-se eficientes em promover efeitos negativos no processo de seleção hospedeira de lagartas $H$. armigera, no mesmo nível que o acesso LA-1401, porém, foram significativamente mais consumidos do

Tabela 3. Número de lagartas às 12, 24, 36 e 48 horas após a liberação de Helicoverpa armigera, e nota visual de área foliar consumida (média \pm erro padrão), 48 horas após a liberação dos insetos, sobre a face abaxial dos folíolos de genótipos de tomateiro, com diferentes densidades de tricomas glandulares do tipo IV (temperatura $25 \pm 2^{\circ} \mathrm{C}$; umidade relativa do ar $70 \pm 2 \%$; e fotófase de 12 horas $)^{(1)}$.

\begin{tabular}{|c|c|c|c|c|c|c|}
\hline \multirow[t]{2}{*}{ Genótipo } & \multirow{2}{*}{$\begin{array}{c}\text { Densidade de } \\
\text { tricomas do tipo IV }\end{array}$} & \multicolumn{4}{|c|}{ Número de lagartas (média \pm erro padrão) } & \multirow{2}{*}{$\begin{array}{c}\text { Nota visual } \\
(\%)\end{array}$} \\
\hline & & 12 horas & 24 horas & 36 horas & 48 horas & \\
\hline 'Santa Clara' & baixa & $3,7 \pm 0,34 a$ & $3,8 \pm 0,28 \mathrm{a}$ & $5,1 \pm 0,28 \mathrm{a}$ & $4,1 \pm 0,51 \mathrm{a}$ & $36,9 \pm 4,35 \mathrm{a}$ \\
\hline TOM-684 & baixa & $3,5 \pm 0,32 \mathrm{a}$ & $3,8 \pm 0,35 \mathrm{a}$ & $4,5 \pm 0,52 \mathrm{a}$ & $3,9 \pm 0,50 \mathrm{a}$ & $37,2 \pm 4,54 \mathrm{a}$ \\
\hline BPX-486-313 & baixa & $3,2 \pm 0,34 \mathrm{a}$ & $3,3 \pm 0,29 a$ & $3,1 \pm 0,34 \mathrm{a}$ & $3,2 \pm 0,28 \mathrm{a}$ & $30,8 \pm 4,39 a$ \\
\hline BPX-486-17 & alta & $1,5 \pm 0,28 b$ & $0,8 \pm 0,23 b$ & $1,6 \pm 0,29 b$ & $0,7 \pm 0,20 b$ & $11,9 \pm 3,05 b$ \\
\hline BPX-486-10 & alta & $1,5 \pm 0,30 \mathrm{~b}$ & $0,4 \pm 0,12 b$ & $1,6 \pm 0,30 b$ & $0,8 \pm 0,20 \mathrm{~b}$ & $6,6 \pm 0,84 b$ \\
\hline BPX-486-46 & alta & $1,5 \pm 0,30 \mathrm{~b}$ & $0,6 \pm 0,19 b$ & $1,6 \pm 0,31 b$ & $1,2 \pm 0,25 b$ & $7,8 \pm 0,72 b$ \\
\hline LA-1401 & alta & $1,2 \pm 0,29 b$ & $1,2 \pm 0,22 b$ & $1,4 \pm 0,20 \mathrm{~b}$ & $0,6 \pm 0,21 b$ & $3,6 \pm 0,63 \mathrm{c}$ \\
\hline \multicolumn{7}{|l|}{ Correlação (r) } \\
\hline \multicolumn{2}{|c|}{ Densidade $\mathrm{x}$ número de lagartas ou nota } & $-0,84^{*}$ & $-0,86^{*}$ & $-0,81^{*}$ & $-0,85^{*}$ & $-0,79 *$ \\
\hline
\end{tabular}


que o acesso selvagem LA-1401, e menos consumidos do que os genótipos com baixa densidade de tricomas glandulares (Tabela 3).

É possível que a diferença entre os genótipos selecionados quanto à alta densidade de tricomas glandulares e o genótipo silvestre esteja associada ao fato de o LA-1401 apresentar maior teor de acilaçúcares não só nos tricomas glandulares do tipo IV, mas também em outras estruturas das folhas, como as células da epiderme foliar (Gonçalves, 2006; Costa, 2013).

Tal como aqui observado, houve menor preferência das lagartas H. armigera de se alimentar de genótipos de tomateiro PT-4287 e 'Seijima Jeisei' (S. lycopersicum) e do híbrido 'Varushanadu' (S. lycopersicum $\mathrm{x}$ $S$. pimpinellifolium) - igualmente associados à alta densidade de tricomas glandulares do tipo VI e maiores teores foliares de ortodihidroxifenóis -, em comparação a uma cultivar comercial de tomateiro (Selvanarayanan $\&$ Narayanasamy, 2006). A antixenose foi observada em lagartas Spodoptera frugiperda (Smith) e S. eridania (Cramer) (Lepidoptera: Noctuidae), em acessos de tomateiro PI 134417, PI 134418 (S. hirsutum var. glabratum), LA-462 (S. peruvianum), PI 126931 (S. pimpinellifolium) e LA-716 (S. pennellii), em que se utilizou como testemunha a cultivar 'Santa Clara' (S. lycopersicum). Os acessos LA-716 e PI 126931, que possuem alta densidade de tricomas foliares do tipo IV, apresentaram os maiores níveis de antixenose a ambas as espécies de lagartas (Souza et al., 2013).

Andrade (2015) investigou genótipos obtidos do cruzamento com o acesso LA-1401 (S. galapagense) quanto à resistência a outro importante inseto-praga do tomateiro, a mosca-branca Bemisia tabaci, e também encontrou alta associação entre a densidade de tricomas glandulares do tipo IV e a antixenose (não preferência) desse inseto.

Em geral, a seleção indireta dos genótipos de tomateiro quanto à alta densidade de tricomas do tipo IV, oriundos do cruzamento $S$. lycopersicum x S. galapagense LA-1401, foi eficiente na obtenção de genótipos com maiores níveis de resistência à lagarta H. armigera, tanto pelo mecanismo de antibiose quanto pelo de antixenose, e pode ser indicada para facilitar o processo de seleção que, quando feita diretamente em campo ou em laboratório, na maioria dos casos, é inviável em grandes populações de plantas, como as segregantes.
Os genótipos da população BPX-486, com alta densidade de tricomas glandulares do tipo IV, são promissores para dar continuidade ao programa de melhoramento do tomateiro, para a obtenção de genótipos comerciais resistentes à lagarta $H$. armigera, por meio de retrocruzamentos, e recuperação das qualidades agronômicas presentes no parental recorrente.

\section{Conclusão}

Os genótipos de tomateiro obtidos do cruzamento interespecífico de Solanum lycopersicum $\mathrm{x}$ $S$. galapagense, selecionados quanto à alta densidade de tricomas glandulares do tipo IV, apresentam resistência à lagarta Helicoverpa armigera, tanto pelo mecanismo de antibiose quanto pelo de antixenose.

\section{Agradecimentos}

Ao Conselho Nacional de Desenvolvimento Científico e Tecnológico (CNPq), à Coordenação de Aperfeiçoamento de Pessoal de Nível Superior (Capes), à Fundação de Amparo à Pesquisa de Minas Gerais (Fapemig), à Universidade Federal de Lavras (Ufla) - em particular aos Departamentos de Biologia, Química, Entomologia e Agricultura -, à Fundação de Apoio ao Ensino, Pesquisa e Extensão de Lavras (Faepe), à Fundação de Desenvolvimento Científico e Cultural (Fundecc) e à Hortiagro Sementes, pelo apoio recebido.

\section{Referências}

ALI, A.; CHOUDHURY, R.A.; AHMAD, Z.; RAHMAN, F.; KHAN, F.R.; AHMAD, S.K. Some biological characteristics of Helicoverpa armigera on chickpea. Tunisian Journal of Plant Protection, v.4, p.99-106, 2009.

ALVARENGA, M.A.R. Tomate: produção em campo, casa de vegetação e hidroponia. 2.ed. rev. e ampl. Lavras: UFLA, 2013. $457 \mathrm{p}$.

ANDRADE, M.C. Genetic control of glandular trichome densities and their association with whitefly resistance from Solanum galapagense accession LA1401. 2015. 96p. Tese (Doutorado) - Universidade Federal de Lavras, Lavras.

BOIÇA JUNIOR, A.L.; FERRAREZZI, R.; RODRIGUES, N.E.L.; SOUZA, B.H.S. de; BOTTEGA, D.B.; SILVA, A.G. da. Resistência de cultivares de amendoim de hábitos de crescimento ereto e rasteiro a Spodoptera cosmioides em laboratório. Revista Agro@ mbiente, v.7, p.80-88, 2013. DOI: 10.18227/1982-8470ragro. v7i1.862. 
COSTA, E. M. R. Relação entre densidades de tricomas foliares e teores de zingibereno e de acilaçúcares em tomateiros resistentes a pragas. 2013. 83p. Tese (Doutorado) - Universidade Federal de Lavras, Lavras.

CZEPAK, C.; ALBERNAZ, K.C.; VIVAN, L.M.; GUIMARÃES, H.O.; CARVALHAIS, T. Primeiro registro de ocorrência de Helicoverpa armigera (Hübner) (Lepidoptera: Noctuidae) no Brasil. Pesquisa Agropecuária Tropical, v.43, p.110-113, 2013. DOI: $10.1590 / \mathrm{S} 1983-40632013000100015$.

DIAS, D.M.; RESENDE, J.T.V.; MARODIN, J.C.; MATOS, R.; LUSTOSA, I.F.; RESENDE, N.C.V. Acyl sugars and whitefly (Bemisia tabaci) resistance in segregating populations of tomato genotypes. Genetics and Molecular Research, v.15, p.1-11, 2016. DOI: $10.4238 /$ gmr.15027788.

FIRDAUS, S.; HEUSDEN, A.W. van; HIDAYATI, N.; SUPENA, E.D.J.; MUMM, R.; VOS, R.C.H. de; VISSER, R.G.F.; VOSMAN, B. Identification and QTL mapping of whitefly resistance components in Solanum galapagense. Theoretical and Applied Genetics, v.126, p.1487-1501, 2013. DOI: 10.1007/ s00122-013-2067-z.

GONÇALVES, L.D. Herança do teor de acilaçúcares em genótipos de tomateiro e sua relação com tricomas foliares e repelência ao ácaro Tetranychus evansi. 2006. 85p. Tese (Doutorado) - Universidade Federal de Lavras, Lavras.

GONÇALVES, L.D.; MALUF, W.R.; CARDOSO, M. das G.; RESENDE, J.T.V. de; CASTRO, E. M. de; SANTOS, N.M.; NASCIMENTO, I.R. do; FARIA, M.V. Relação entre zingibereno, tricomas foliares e repelência de tomateiros a Tetranychus evansi. Pesquisa Agropecuária Brasileira, v.41, p.267-273, 2006. DOI: 10.1590/S0100-204X2006000200011.

GREENE, G.L.; LEPPLA, N.C.; DICKERSON, W.A. Velvetbean caterpillar: a rearing procedure and artificial medium. Journal of Economic Entomology, v.69, p.487-488, 1976. DOI: 10.1093/ jee/69.4.487.

JOUY, N.; BORDAT, D.; BESSIÈRE, J.M. Identification of (2,3,4-tri-O-acyl)-a-D-glucopyranosil-(3-O-acyl)-b-D-fructofuranoside), responsible of the high level of leafminer resistance in Lycopersicon cheesmanii. Report of the Tomato Genetics Cooperative, v.42, p.22, 1992.

KRAUS, J.E.; ARDUIM, M. Manual básico de métodos em morfologia vegetal. Seropédica: EDUR, 1997. 221p.

LUCATTI, A. -F.; HEUSDEN, A. -W. van; VOS, R.C.H. de; VISSER, R.G.F.; VOSMAN, B. Differences in insect resistance between tomato species endemic to the Galapagos Islands. BMC Evolutionary Biology, v.13, article 175, 2013. DOI: 10.1186/1471-2148-13-175.

LUCINI, T.; FARIA, M.V.; ROHDE, C.; RESENDE, J.T.V.; OLIVEIRA, J.R.F. de. Acylsugar and the role of trichomes in tomato genotypes resistance to Tetranychus urticae. Arthropod-Plant Interactions, v.9, p.45-53, 2015. DOI: 10.1007/ s11829-014-9347-7.

LUCKWILL, L.C. The genus Lycopersicon: an historical, biological, and taxonomic survey of the wild and cultivated tomatoes. Aberdeen: Aberdeen University Press, 1943. 44p.
MALUF, W.R.; SILVA, V. de F.; CARDOSO, M. das G.; GOMES, L.A.A.; GONÇALVES NETO, A.C.; MACIEL, G.M.; NÍZIO, D.A.C. Resistance to the South American tomato pinworm Tuta absoluta in high acylsugar and/or high zingiberene tomato genotypes. Euphytica, v.176, p.113-123, 2010. DOI: 10.1007/ s10681-010-0234-8.

MAMTA, K.; REDDY, R.K.; RAJAM, M.V. Targeting chitinase gene of Helicoverpa armigera by host-induced RNA interference confers insect resistance in tobacco and tomato. Plant Molecular Biology, v.90, p.281-292, 2016. DOI: 10.1007/s11103-015-0414-y.

MOREIRA, L.A.; PICANÇO, M.C.; SILVA, G.A.; SEMEÃO, A.A.; CASALI, V.W.D.; CAMPOS, M.R. de; FERNANDES, M.E. de S.; XAVIER, V.M. Antibiosis of eight Lycopersicon genotypes to Tuta absoluta (Lepidoptera: Gelechiidae). Revista Ceres, v.56, p.283-287, 2009.

MUTHUKUMARAN, N. Biophysical and biochemical factors of resistance in tomato accessions as influenced by selected bioinoculants against fruit worm Helicoverpa armigera (Hübner). International Journal of Current Microbiology and Applied Sciences, v.5, p.252-262, 2016. DOI: 10.20546/ ijcmas.2016.501.024.

PRATISSOLI, D.; LIMA, V.L.S.; PIROVANI, V.D.; LIMA, W.L. Occurrence of Helicoverpa armigera (Lepidoptera: Noctuidae) on tomato in the Espírito Santo state. Horticultura Brasileira, v.33, p.101-105, 2015. DOI: 10.1590/S0102-053620150000100016.

SAFURAIE-PARIZI, S.; FATHIPOUR, Y.; TALEBI, A.A. Evaluation of tomato cultivars to Helicoverpa armigera using two-sex life Table parameters in laboratory. Journal of Asia-Pacific Entomology, v.17, p.837-844, 2014. DOI: 10.1016/j. aspen.2014.08.004.

SELVANARAYANAN, V.; NARAYANASAMY, P. Factors of resistance in tomato accessions against the fruit worm, Helicoverpa armigera (Hubner). Crop Protection, v.25, p.1075-1079, 2006. DOI: 10.1016/j.cropro.2006.02.008.

SILVA, V. de F.; MALUF, W.R.; CARDOSO, M. das G.; GONÇALVES NETO, A.C.; MACIEL, G.M.; NÍZIO, D.A.C.; SILVA, V.A. Resistência mediada por aleloquímicos de genótipos de tomateiro à mosca-branca e ao ácaro-rajado. Pesquisa Agropecuária Brasileira, v.44, p.1262-1269, 2009. DOI: 10.1590/ S0100-204X2009001000008.

SIMMONS, A.T.; GURR, G.M. Trichomes of Lycopersicon species and their hybrids: effects on pests and natural enemies. Agricultural and Forest Entomology, v.7, p.265-276, 2005. DOI: 10.1111/j.1461-9555.2005.00271.x.

SIMMONS, A.T.; GURR, G.M.; MCGRATH, D.; MARTIN, P.M.; NICOL, H.I. Entrapment of Helicoverpa armigera (Hübner) (Lepidoptera: Noctuidae) on glandular trichomes of Lycopersicon species. Australian Journal of Entomology, v.43, p.196-200, 2004. DOI: 10.1111/j.1440-6055.2004.00414.x.

SOUZA, B.H.S. de; BOTTEGA, D.B.; SILVA, A.G. da; BOIÇA JÚNIOR, A.L. Feeding non-preference by Spodoptera frugiperda and Spodoptera eridania on tomato genotypes. Revista Ceres, v.60, p.21-29, 2013. DOI: 10.1590/S0034-737X2013000100004.

SPECHT, A.; SOSA-GÓMEZ, D.R.; PAULA-MORAES, S.V. de; YANO, S.A.C. Identificação morfológica e molecular de 
Helicoverpa armigera (Lepidoptera: Noctuidae) e ampliação de seu registro de ocorrência no Brasil. Pesquisa Agropecuária Brasileira, v.48, p.689-692, 2013. DOI: 10.1590/S0100204X2013000600015.

TALEKAR, N.S.; OPEÑA, R.T.; HANSON, P. Helicoverpa armigera management: a review of AVRDC's research on host plant resistance in tomato. Crop Protection, v.25, p.461-467, 2006. DOI: 10.1016/j.cropro.2005.07.011.
TIAN, D.; TOOKER, J.; PEIFFER, M.; CHUNG, S.H.; FELTON, G.W. Role of trichomes in defense against herbivores: comparison of herbivore response to woolly and hairless trichome mutants in tomato (Solanum lycopersicum). Planta, v.236, p.1053-1066, 2012.

USMAN, A.; KHAN, I.A.; INAYATULLAH, M.; SALJOQI, A.U.R.; SHAH, M. Appraisal of different tomato genotypes against tomato fruit worm (Helicoverpa armigera Hub.) infestation. Pakistan Journal of Zoology, v.45, p.113-119, 2013.

Recebido em $1^{\circ}$ de março de 2016 e aprovado em 18 de maio de 2016 